\title{
ENUMERATING BRANCHED SURFACE COVERINGS FROM UNBRANCHED ONES
}

\author{
JIN HO KWAK, JAEUN LEE AND ALEXANDER MEDNYKH
}

\begin{abstract}
The number of non-isomorphic $n$-fold branched coverings of a given closed surface can be determined by the number of nonisomorphic $n$-fold unbranched coverings of the surface and the number of nonisomorphic connected $n$-fold graph coverings of a suitable bouquet of circles. A similar enumeration can also be done for regular branched coverings. Some explicit enumerations are also possible.
\end{abstract}

\section{Introduction}

Throughout this paper, a surface $\mathbb{S}$ means a compact connected 2-manifold without boundary. By the classification theorem of surfaces, a surface $\mathbb{S}$ is homeomorphic to one of the following:

$$
\mathbb{S}_{k}= \begin{cases}\text { the orientable surface with } k \text { handles, } & \text { if } k \geqslant 0, \\ \text { the nonorientable surface with }-k \text { crosscaps, } & \text { if } k<0 .\end{cases}
$$

A continuous function $p: \tilde{\mathbb{S}} \rightarrow \mathbb{S}$ from a surface $\tilde{\mathbb{S}}$ onto another surface $\mathbb{S}$ is called a branched covering if there exists a finite set $B$ in $\mathbb{S}$ such that the restriction of $p$ to $\tilde{\mathbb{S}}-p^{-1}(B),\left.p\right|_{\tilde{S}-p^{-1}(B)}: \tilde{\mathbb{S}}-p^{-1}(B) \rightarrow \mathbb{S}-B$, is a covering projection in the usual sense. The smallest subset $B$ of $\mathbb{S}$ that has this property is called the branch set.

A branched covering $p: \widetilde{\mathbb{S}} \rightarrow \mathbb{S}$ is regular if there exists a (finite) group $\mathcal{A}$ that acts on $\tilde{\mathbb{S}}$ with at most finitely many fixed points, so that the surface $\mathbb{S}$ is homeomorphic to the quotient space $\tilde{\mathbb{S}} / \mathcal{A}$, say by $h$, and the quotient map $\tilde{\mathbb{S}} \rightarrow \tilde{\mathbb{S}} / \mathcal{A}$ is the composition $h \circ p$ of $p$ and $h$. We call it simply a branched $\mathcal{A}$-covering. In this case, the group $\mathcal{A}$ becomes the covering transformation group of the branched covering $p: \widetilde{\mathbb{S}} \rightarrow \mathbb{S}$. Two branched coverings $p: \tilde{\mathbb{S}} \rightarrow \mathbb{S}$ and $q: \tilde{\mathbb{S}}^{\prime} \rightarrow \mathbb{S}$ are isomorphic if there exists a homeomorphism $h: \tilde{\mathbb{S}} \rightarrow \tilde{\mathbb{S}}^{\prime}$ such that $p=q \circ h$.

Recently, Kwak et al. $[\mathbf{1 2}, \mathbf{1 4}]$ examined the conditions under which a surface can be a branched $\mathscr{A}$-covering of a given surface with a given branch set, when $\mathscr{A}$ is the cyclic group $\mathbb{Z}_{p}$ or the dihedral group $\mathbb{D}_{p}$ of order $2 p$, for $p$ a prime.

In this paper, we derive an enumeration formula for the total number of non-isomorphic (regular) branched coverings of any given surface $\mathbb{S}$ with branch set $B$, in terms of the nonisomorphic unbranched coverings and some non-isomorphic graph coverings of a suitable bouquet of circles.

First author supported by $\mathrm{Com}^{2} \mathrm{MaC}-\mathrm{KOSEF}$; second author supported by KRF(2000-015-DP0037); third author supported by RFBR(99-01-00630).

Received 31 July 2001, revised 14 November 2002; published 2 June 2003.

2000 Mathematics Subject Classification 05C10, 05C30, 57M12

(C) 2003, Jin Ho Kwak, Jaeun Lee and Alexander Mednykh 


\section{A classification of branched coverings}

Let $G$ be a finite connected graph with vertex set $V(G)$ and edge set $E(G)$. We allow loops and multiple edges. Notice that $G$ can be identified with a one-dimensional CW complex in the Euclidean 3 -space $\mathbb{R}^{3}$, so that every graph map is continuous. Every covering of a graph $G$ can be constructed as follows (see [3]).

Every edge of a graph $G$ gives rise to a pair of oppositely directed edges. By $e^{-1}=v u$, we mean the reverse edge to a directed edge $e=u v$. We denote the set of directed edges of $G$ by $D(G)$. Each directed edge $e$ has an initial vertex $i_{e}$ and a terminal vertex $t_{e}$. Following [3], a permutation voltage assignment $\phi$ on a graph $G$ is a map $\phi: D(G) \rightarrow S_{n}$ with the property that $\phi\left(e^{-1}\right)=\phi(e)^{-1}$ for each $e \in D(G)$, where $S_{n}$ is the symmetric group on $n$ elements $\{1,2, \ldots, n\}$. The permutation derived graph $G^{\phi}$ is defined as follows: $V\left(G^{\phi}\right)=V(G) \times\{1,2, \ldots, n\}$, and for each edge $e \in D(G)$ and $j \in\{1,2, \ldots, n\}$ there is an edge $(e, j)$ in $D\left(G^{\phi}\right)$ with $i_{(e, j)}=\left(i_{e}, j\right)$ and $t_{(e, j)}=\left(t_{e}, \phi(e) j\right)$. The natural projection $p_{\phi}: G^{\phi} \rightarrow G$ is a covering. In the derived graph $G^{\phi}$, a vertex $(u, i)$ is denoted by $u_{i}$, and an edge $(e, j)$ is denoted by by $e_{j}$. Let $\mathcal{A}$ be a finite group. An ordinary voltage assignment (or, A-voltage assignment) of $G$ is a function $\phi: D(G) \rightarrow \mathcal{A}$ with the property that $\phi\left(e^{-1}\right)=\phi(e)^{-1}$ for each $e \in D(G)$. The values of $\phi$ are called voltages, and $\mathcal{A}$ is called the voltage group. The ordinary derived graph $G \times \times_{\phi} \mathcal{A}$ has as its vertex set $V(G) \times \mathcal{A}$ and as its edge set $E(G) \times \mathcal{A}$, so that an edge $(e, g)$ of $G \times_{\phi} \mathcal{A}$ joins a vertex $(u, g)$ to $(v, \phi(e) g)$ for $e=u v \in D(G)$ and $g \in \mathcal{A}$. In the ordinary derived graph $G \times_{\phi} \mathcal{A}$, a vertex $(u, g)$ and an edge $(e, g)$ are denoted by $u_{g}$ and $e_{g}$, respectively. The first coordinate projection $p_{\phi}: G \times_{\phi} \mathcal{A} \rightarrow G$, called the natural projection, commutes with the left multiplication action of the $\phi(e)$ and the right action of $\mathcal{A}$ on the fibers, which is free and transitive, so that $p$ is a regular $|\mathcal{A}|$-fold covering, called simply an $\mathcal{A}$-covering.

A (branched) surface covering is closely related to a graph covering that is embeddable into it. To see such a relation, we first review a graph embedding into a surface.

An embedding of a graph $G$ into a surface $\mathbb{S}$ is a continuous one-to-one function $l$ : $G \rightarrow \mathbb{S}$. If every component of $\mathbb{S}-\imath(G)$, called a region, is homeomorphic to an open disk, then $\imath: G \rightarrow \mathbb{S}$ is called a 2-cell embedding. Such an embedding can be described by a combinatorial method as follows. An embedding scheme $(\rho, \lambda)$ for a graph $G$ consists of a rotation scheme $\rho$, which assigns a cyclic permutation $\rho_{v}$ on $N(v)=\{e \in D(G)$ : the initial vertex of $e$ is $v\}$ to each $v \in V(G)$, and a voltage assignment $\lambda$, which assigns a value $\lambda(e)$ in $\mathbb{Z}_{2}=\{-1,1\}$ to each $e \in E(G)$.

It is well known that every embedding scheme determines a 2-cell embedding of $G$ into an orientable or non-orientable surface $\mathbb{S}$; also, every 2-cell embedding of $G$ into a surface $\mathbb{S}$ is determined by such a scheme (see [19] or [20]).

Let $\imath: G \rightarrow \mathbb{S}$ be a 2-cell embedding with embedding scheme $(\rho, \lambda)$, and let $\phi$ be a permutation voltage assignment. The derived graph $G^{\phi}$ has the derived embedding scheme $(\tilde{\rho}, \tilde{\lambda})$, which is defined by $\tilde{\rho}_{v_{i}}\left(e_{i}\right)=\left(\rho_{v}(e)\right)_{i}$ and $\tilde{\lambda}\left(e_{i}\right)=\lambda(e)$ for each $e_{i} \in D\left(G^{\phi}\right)$. Then it induces a 2 -cell embedding of $G^{\phi}$ into a surface, say $\tilde{\imath}: G^{\phi} \rightarrow \mathbb{S}^{\phi}$, such that the following diagram commutes.

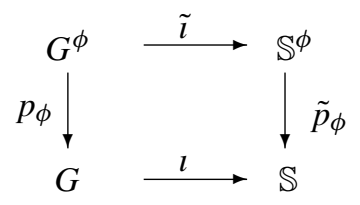




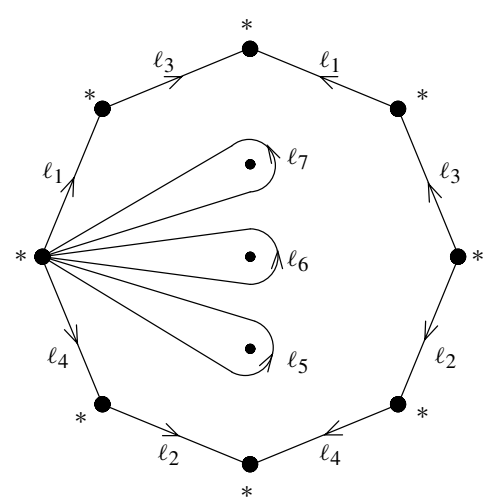

(a) $\mathfrak{B}_{7} \hookrightarrow \mathbb{S}_{2}-B$

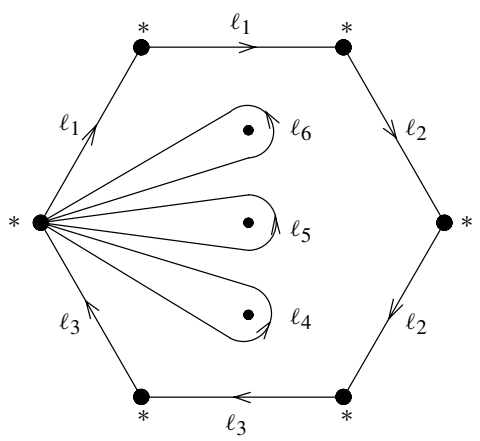

(b) $\mathfrak{B}_{6} \hookrightarrow \mathbb{S}_{-3}-B$

Figure 1: Two examples of standard embeddings

Moreover, if $G^{\phi}$ is connected, then $\mathbb{S}^{\phi}$ is connected and $\tilde{p}_{\phi}: \mathbb{S}^{\phi} \rightarrow \mathbb{S}$ is a covering, possibly having branch points. Conversely, let $p: \tilde{\mathbb{S}} \rightarrow \mathbb{S}$ be a branched $n$-fold covering of a surface $\mathbb{S}$. Then there exists a 2-cell embedding $\imath: G \rightarrow \mathbb{S}$ of a graph $G$ such that each face of the embedding has at most one branch point interior to it, and a permutation voltage assignment $\phi: D(G) \rightarrow S_{n}$ such that the branched $n$-fold covering $\tilde{p}_{\phi}: \mathbb{S}^{\phi} \rightarrow \mathbb{S}$ is isomorphic to the given branched covering $p: \widetilde{\mathbb{S}} \rightarrow \mathbb{S}$; see [4].

A surface $\mathbb{S}_{k}$ can be represented by: (i) a $4 k$-gon with identification data $\prod_{s=1}^{k} a_{s} b_{s} a_{s}^{-1} b_{s}^{-1}$ on its boundary if $k>0$; (ii) a $-2 k$-gon with identification data $\prod_{s=1}^{-k} a_{s} a_{s}$ on its boundary if $k<0$; and (iii) a bigon with identification data $a a^{-1}$ on its boundary if $k=0$.

Let $B$ be a finite set of points in $\mathbb{S}_{k}$. Note that the fundamental group $\pi_{1}\left(\mathbb{S}_{k}-B, *\right)$ of the punctured surface $\mathbb{S}_{k}-B$ with the base point $* \in \mathbb{S}_{k}-B$ can be represented by

$$
\begin{array}{ll}
\left\langle a_{1}, \ldots, a_{k}, b_{1}, \ldots, b_{k}, c_{1}, \ldots, c_{|B|} ; \prod_{s=1}^{k} a_{s} b_{s} a_{s}^{-1} b_{s}^{-1} \prod_{t=1}^{|B|} c_{t}=1\right\rangle, & \text { if } k>0 ; \\
\left\langle a_{1}, \ldots, a_{-k}, c_{1}, \ldots, c_{|B|} ; \prod_{s=1}^{-k} a_{s} a_{s} \prod_{t=1}^{|B|} c_{t}=1\right\rangle, & \text { if } k<0 ; \\
\left\langle c_{1}, \ldots, c_{|B|} ; \prod_{t=1}^{|B|} c_{t}=1\right\rangle, & \text { if } k=0 .
\end{array}
$$

We call this the standard presentation of the fundamental group $\pi_{1}\left(\mathbb{S}_{k}-B, *\right)$. For each $t=1,2, \ldots,|B|$, we take a simple closed curve based at $*$ lying in the face determined by the polygonal representation of the surface $\mathbb{S}_{k}$ so that it represents the homotopy class of the generator $c_{t}$. Then it induces a 2-cell embedding of a bouquet of $m$ circles, say $\mathfrak{B}_{m}$, into the surface $\mathbb{S}_{k}$ such that the embedding has: (i) $|B| 1$-sided regions and one $(|B|+4 k)$-sided region if $k>0$; (ii) $|B| 1$-sided regions and one $(|B|-2 k)$-sided region if $k<0$; and (iii) $|B| 1$-sided regions and one $|B|$-sided region if $k=0$, where $m$ is the number of the generators of the standard presentation of the corresponding fundamental group. This embedding $\imath: \mathfrak{B}_{m} \rightarrow \mathbb{S}_{k}$ is called the standard embedding, simply denoted by $\mathfrak{B}_{m} \hookrightarrow \mathbb{S}_{k}-B$. For example, Figure 1 illustrates the standard embeddings of bouquets with $|B|=3$. Figure 1(a) represents the standard embedding $\mathfrak{B}_{7} \hookrightarrow \mathbb{S}_{2}-B$, and Figure 1(b) shows the standard embedding $\mathfrak{B}_{6} \hookrightarrow \mathbb{S}_{-3}-B$. 
For a natural number $n$, let $C^{1}\left(\mathfrak{B}_{m} ; n\right)$ denote the set of all permutation voltage assignments $\phi: D\left(\mathfrak{B}_{m}\right) \rightarrow S_{n}$ on the bouquet of $m$ circles $\mathfrak{B}_{m}$. Notice that $C^{1}\left(\mathfrak{B}_{m} ; n\right)$ can be identified with the cartesian product $\left(S_{n}\right)^{m}$ of $m$ copies of the symmetric group $S_{n}$; that is, each element $\phi$ in $C^{1}\left(\mathfrak{B}_{m} ; n\right)$ can be identified with an $m$-tuple $\left(\phi\left(\ell_{1}\right), \ldots, \phi\left(\ell_{m}\right)\right)$, where $\ell_{i}$ is a positively oriented loop in $D\left(\mathfrak{B}_{m}\right)$. For convenience, let $a_{k}=2 k$ if $k \geqslant 0$, and let $a_{k}=-k$ if $k<0$. Let $C^{1}\left(\mathfrak{B}_{a_{k}+|B|} \hookrightarrow \mathbb{S}_{k}-B ; n\right)$ and $C^{1}\left(\mathfrak{B}_{a_{k}+|B|} \hookrightarrow \mathbb{S}_{k}-B ; \mathcal{A}\right)$ respectively denote the subset of $\left(S_{n}\right)^{a_{k}+|B|}$ and the subset of $(\mathcal{A})^{a_{k}+|B|}$ consisting of all $\left(a_{k}+|B|\right)$-tuples $\left(\sigma_{1}, \ldots, \sigma_{a_{k}+|B|}\right)$ that satisfy the following three conditions.

(C1) The subgroup $\left\langle\sigma_{1}, \ldots, \sigma_{a_{k}+|B|}\right\rangle$ generated by $\left\{\sigma_{1}, \ldots, \sigma_{a_{k}+|B|}\right\}$ is transitive on $\{1,2, \ldots, n\}$, or else is the full group $\mathcal{A}$.

(C2) (i) If $k \geqslant 0$, then

$$
\prod_{i=1}^{k} \sigma_{i} \sigma_{k+i} \sigma_{i}^{-1} \sigma_{k+i}^{-1} \prod_{i=1}^{|B|} \sigma_{a_{k}+i}=1
$$

(ii) if $k<0$, then

$$
\prod_{i=1}^{-k} \sigma_{i} \sigma_{i} \prod_{i=1}^{|B|} \sigma_{a_{k}+i}=1 .
$$

(C3) $\sigma_{i} \neq 1$ for each $i=a_{k}+1, \ldots, a_{k}+|B|$.

Note that condition (C1) guarantees that the surface $\mathbb{S}^{\phi}$ is connected, and conditions (C2) and (C3) ensure that the set $B$ is the same as the branch set of the branched covering $\tilde{p}_{\phi}: \mathbb{S}^{\phi} \rightarrow \mathbb{S}$. By using a method similar to that given in [14], one can obtain the following theorem.

THEOREM 1 (EXISTENCE AND CLASSIFICATION OF BRANCHED COVERINGS). Every permutation voltage assignment in $C^{1}\left(\mathfrak{B}_{a_{k}+|B|} \hookrightarrow \mathbb{S}_{k}-B ; n\right)$ induces a connected branched $n$-fold covering of $\mathbb{S}_{k}$ with branch set $B$. Conversely, every connected branched $n$-fold covering of $\mathbb{S}_{k}$ with branch set $B$ can be derived from a voltage assignment in $C^{1}\left(\mathfrak{B}_{a_{k}+|B|} \hookrightarrow \mathbb{S}_{k}-B ; n\right)$. Moreover, for any two permutation voltage assignments $\phi, \psi \in C^{1}\left(\mathfrak{B}_{a_{k}+|B|} \hookrightarrow \mathbb{S}-B ; n\right)$, two branched $n$-fold surface coverings $\tilde{p}_{\phi}: \mathbb{S}^{\phi} \rightarrow \mathbb{S}$ and $\tilde{p}_{\psi}: \mathbb{S}^{\psi} \rightarrow \mathbb{S}$ are isomorphic if and only if two graph coverings $p_{\phi}: \mathfrak{B}_{a_{k}+|B|}^{\phi} \rightarrow \mathfrak{B}_{a_{k}+|B|}$ and $p_{\psi}: \mathfrak{B}_{a_{k}+|B|}^{\psi} \rightarrow \mathfrak{B}_{a_{k}+|B|}$ are isomorphic. Equivalently, there exists a permutation $\sigma \in S_{n}$ such that

$$
\psi\left(\ell_{i}\right)=\sigma \phi\left(\ell_{i}\right) \sigma^{-1}
$$

for all $\ell_{i} \in D\left(\mathfrak{B}_{a_{k}+|B|}\right)$, where $a_{k}=2 k$ if $k \geqslant 0$, and $a_{k}=-k$ if $k<0$.

For a finite group $\mathcal{A}$, let $S_{\mathcal{A}}$ denote the symmetric group on the group elements of $\mathcal{A}$. It gives the (left) regular representation $\mathcal{A} \rightarrow \mathcal{\wp}_{\mathcal{A}}$ of $\mathcal{A}$ via $g \rightarrow L(g)$, the left translation by $g$ on $\mathcal{A}$. Clearly, this representation is faithful and the group $\mathcal{A}$ can be identified with the group of left translations $L(g): \mathcal{A} \equiv\{L(g) \mid g \in \mathcal{A}\}$ (Cayley theorem). Notice that a permutation voltage assignment $\phi: D(G) \rightarrow S_{\mathcal{A}}$ having its images in $\mathcal{A}$ can be considered as an $\mathcal{A}$-voltage assignment of $G$, and for such a voltage assignment $\phi$, the permutation derived graph $G^{\phi}$ is nothing but the ordinary derived graph $G \times_{\phi} \mathcal{A}$. By using this fact, Corollary 1 was shown in [14]. 
COROLlARY 1 (EXISTENCE \& CLASSIFICATION OF REGULAR BRANCHED COVERINGS). Every ordinary voltage assignment in $C^{1}\left(\mathfrak{B}_{a_{k}+|B|} \hookrightarrow \mathbb{S}_{k}-B ; \mathcal{A}\right)$ induces a connected branched $\mathcal{A}$-covering of $\mathbb{S}_{k}$ with branch set $B$.

Conversely, every connected branched A-covering of $\mathbb{S}_{k}$ with branch set B can be derived from a voltage assignment in $C^{1}\left(\mathfrak{B}_{a_{k}+|B|} \hookrightarrow \mathbb{S}_{k}-B ; \mathcal{A}\right)$. Moreover, for any two voltage assignments $\phi, \psi \in C^{1}\left(\mathfrak{B}_{a_{k}+|B|} \hookrightarrow \mathbb{S}-B ; \mathcal{A}\right)$, two branched $\mathcal{A}$-coverings $\tilde{p}_{\phi}: \mathbb{S}^{\phi} \rightarrow \mathbb{S}$ and $\tilde{p}_{\psi}: \mathbb{S}^{\psi} \rightarrow \mathbb{S}$ are isomorphic if and only if two graph coverings $p_{\phi}: \mathfrak{B}_{a_{k}+|B|} \times_{\phi} \mathcal{A} \rightarrow \mathfrak{B}_{a_{k}+|B|}$ and $p_{\psi}: \mathfrak{B}_{a_{k}+|B|} \times_{\psi} \mathcal{A} \rightarrow \mathfrak{B}_{a_{k}+|B|}$ are isomorphic. It is also equivalent to say that there exists a group automorphism $\sigma$ of $\mathcal{A}$ such that

$$
\psi\left(\ell_{i}\right)=\sigma\left(\phi\left(\ell_{i}\right)\right)
$$

for all $\ell_{i} \in D\left(\mathfrak{B}_{a_{k}+|B|}\right)$, where $a_{k}=2 k$ if $k \geqslant 0$, and $a_{k}=-k$ if $k<0$.

\section{Computational formulas}

To derive an enumeration formula for the isomorphism classes of branched surface coverings, we define an $S_{n}$-action on the set $C^{1}\left(\mathfrak{B}_{m} ; n\right)=\left(S_{n}\right)^{m}$ by a simultaneously coordinatewise conjugation; that is, for any $g \in S_{n}$ and any $\left(\sigma_{1}, \ldots, \sigma_{m}\right) \in C^{1}\left(\mathfrak{B}_{m} ; n\right)$,

$$
g \cdot\left(\sigma_{1}, \ldots, \sigma_{m}\right)=\left(g \sigma_{1} g^{-1}, \ldots, g \sigma_{m} g^{-1}\right) .
$$

It follows from Theorem 1 that two voltage assignments in $C^{1}\left(\mathfrak{B}_{a_{k}+|B|} \hookrightarrow \mathbb{S}_{k}-B ; n\right)$ derive isomorphic branched coverings of $\mathbb{S}_{k}$ if and only if they belong to the same orbit under the $S_{n}$-action. Hence we have the following lemma.

Lemma 1. Let $k$ be any integer, and let $B$ be a finite subset of the surface $\mathbb{S}_{k}$. Then the number of non-isomorphic connected $n$-fold branched coverings of the surface $\mathbb{S}_{k}$ with branch set $B$ is

$$
\operatorname{Isoc}\left(\mathbb{S}_{k}, B ; n\right)=:\left|C^{1}\left(\mathfrak{B}_{a_{k}+|B|} \hookrightarrow \mathbb{S}_{k}-B ; n\right) / S_{n}\right|
$$

Now we aim to express the number $\operatorname{Isoc}\left(\mathbb{S}_{k}, B ; n\right)$ in terms of known parameters. Let $\mathfrak{C}\left(\mathfrak{B}_{m} ; n\right)$ denote the set of all $m$-tuples $\left(\sigma_{1}, \ldots, \sigma_{m}\right)$ in $\left(S_{n}\right)^{m}$ such that the group $\left\langle\sigma_{1}, \ldots, \sigma_{m}\right\rangle$ generated by $\left\{\sigma_{1}, \ldots, \sigma_{m}\right\}$ is transitive on $\{1,2, \ldots, n\}$; that is,

$$
\mathfrak{C}\left(\mathfrak{B}_{m} ; n\right)=\left\{\left(\sigma_{1}, \sigma_{2}, \ldots, \sigma_{m}\right) \in\left(S_{n}\right)^{m}:\left\langle\sigma_{1}, \sigma_{2}, \ldots, \sigma_{m}\right\rangle \text { is transitive on }\{1,2, \ldots, n\}\right\} \text {. }
$$

Then $\mathfrak{C}\left(\mathfrak{B}_{m} ; n\right)$ contains all the representatives of the connected $n$-fold coverings of the bouquet of $m$-circles $\mathfrak{B}_{m}$, and the number $\operatorname{Isoc}\left(\mathfrak{B}_{m} ; n\right)$ of non-isomorphic connected $n$ fold coverings of $\mathfrak{B}_{m}$ is equal to $\left|\mathfrak{C}\left(\mathfrak{B}_{m} ; n\right) / S_{n}\right|$, where the $S_{n}$-action on $\mathfrak{C}\left(\mathfrak{B}_{m} ; n\right)$ is also defined by the simultaneously coordinatewise conjugation (see $[10,11]$ ).

Lemma 2. Let $k$ be an integer, and let $b$ be a nonnegative integer. For each $0 \leqslant t \leqslant b$, let

$$
\begin{aligned}
& S(k, b, t) \\
& \quad=\left\{\phi \in\left(S_{n}\right)^{a_{k}+b}: \phi \text { satisfies }(\mathrm{C} 1) \text { and }(\mathrm{C} 2) \text {, and } \sigma_{i}=1, \forall i=a_{k}+1, \ldots, a_{k}+t\right\},
\end{aligned}
$$

where $\phi=\left(\sigma_{1}, \sigma_{2}, \ldots, \sigma_{a_{k}+b}\right)$. If $t=b$, then the set $S(k, b, b)$ is equal to the set $C^{1}\left(\mathfrak{B}_{a_{k}} \hookrightarrow \mathbb{S}_{k} ; n\right)$, and if $t \neq b$, then there is a one-to-one correspondence between the sets $S(k, b, t)$ and $\mathfrak{C}\left(\mathfrak{B}_{a_{k}+b-t-1} ; n\right)$. Moreover, the correspondence preserves the $S_{n}$-action on both the sets, which are defined by simultaneously coordinatewise conjugacy. 
Proof. The case where $t=b$ is clear. Assume that $t \neq b$. Then every element in $S(k, b, t)$ is of the form $\left(\sigma_{1}, \ldots, \sigma_{a_{k}}, 1, \ldots, 1, \sigma_{a_{k}+t+1}, \ldots, \sigma_{a_{k}+b}\right)$. It follows from conditions (C1) and (C2) that the function $f: S(k, b, t) \rightarrow \mathfrak{C}\left(\mathfrak{B}_{a_{k}+b-t-1} ; n\right)$ defined by

$$
f\left(\sigma_{1}, \ldots, \sigma_{a_{k}}, 1, \ldots, 1, \sigma_{a_{k}+t+1}, \ldots, \sigma_{a_{k}+b}\right)=\left(\sigma_{1}, \ldots, \sigma_{a_{k}}, \sigma_{a_{k}+t+1}, \ldots, \sigma_{a_{k}+b-1}\right)
$$

is well-defined and bijective. (Note that the function $f$ is defined by deleting 1 's and the last coordinate.) This completes the proof.

The following is one of the main theorems in this paper.

THEOREM 2. Let $k$ be any integer, and let $B$ be a b-subset of the surface $\mathbb{S}_{k}$. Then the number of connected $n$-fold branched coverings of the surface $\mathbb{S}_{k}$ with branch set $B$ is

$$
\operatorname{Isoc}\left(\mathbb{S}_{k}, B ; n\right)=(-1)^{b} \operatorname{Isoc}\left(\mathbb{S}_{k}, \emptyset ; n\right)+\sum_{t=0}^{b-1}(-1)^{t}\left(\begin{array}{l}
b \\
t
\end{array}\right) \operatorname{Isoc}\left(\mathfrak{B}_{a_{k}+b-t-1} ; n\right),
$$

where $\mathfrak{B}_{m}$ is a bouquet of $m$ circles, $a_{k}=2 k$ if $k \geqslant 0$, and $a_{k}=-k$ if $k<0$.

Proof. For each $i=a_{k}+1, \ldots, a_{k}+b$, let $\mathcal{P}_{i}$ be the property that the $i$ th coordinate of an element of $\left(S_{n}\right)^{a_{k}+b}$ is the identity. For each subset $S$ of $\left\{a_{k}+1, \ldots, a_{k}+b\right\}$, let $N\left(\mathcal{P}_{S}\right)$ be the number of elements in the product $\left(S_{n}\right)^{a_{k}+b}$ that satisfy conditions (C1) and (C2) and the properties $\mathcal{P}_{i}$ for all $i \in S$. Notice that $N\left(\mathcal{P}_{\emptyset}\right)$ is the number of all elements in the product $\left(S_{n}\right)^{a_{k}+b}$ that satisfy not only conditions $(\mathrm{C} 1)$ and $(\mathrm{C} 2)$, but also the requirement that the set $C^{1}\left(\mathfrak{B}_{a_{k}+b} \hookrightarrow \mathbb{S}_{k}-B ; n\right)$ be equal to the set of elements of $\left(S_{n}\right)^{a_{k}+b}$ that satisfy conditions (C1) and (C2), but not any other property $\mathcal{P}_{i}$ for $i=a_{k}+1, \ldots, a_{k}+b$. It follows from the inclusion-exclusion principle that

$$
\left|C^{1}\left(\mathfrak{B}_{a_{k}+b} \hookrightarrow \mathbb{S}_{k}-B ; n\right)\right|=\sum_{t=0}^{b}(-1)^{t}\left(\sum_{\substack{S \subset\left\{a_{k}+1, \ldots, a_{k}+b\right\} \\|S|=t}} N\left(\mathcal{P}_{S}\right)\right) .
$$

Since $N\left(\mathcal{P}_{S}\right)=N\left(\mathcal{P}_{S^{\prime}}\right)$ for any two subsets $S$ and $S^{\prime}$ of $\left\{a_{k}+1, \ldots, a_{k}+b\right\}$ with the same cardinality, we have

$$
\begin{aligned}
& \sum_{S \subset\left\{a_{k}+1, \ldots, a_{k}+b\right\},|S|=t} N\left(\mathcal{P}_{S}\right) \\
& =\left(\begin{array}{l}
b \\
t
\end{array}\right) \mid\left\{\phi \in\left(S_{n}\right)^{a_{k}+b}: \phi \text { satisfies }(\mathrm{C} 1) \text { and }(\mathrm{C} 2) \text {, and } \sigma_{i}=1, \forall i=a_{k}+1, \ldots, a_{k}+t\right\} \mid .
\end{aligned}
$$

Now, from Lemma 2, we have

$$
\begin{aligned}
\left|C^{1}\left(\mathfrak{B}_{a_{k}+b} \hookrightarrow \mathbb{S}_{k}-B ; n\right)\right| & \\
& =\sum_{t=0}^{b-1}(-1)^{t}\left(\begin{array}{c}
b \\
t
\end{array}\right)\left|\mathfrak{C}\left(\mathfrak{B}_{a_{k}+b-t-1} ; n\right)\right|+(-1)^{b}\left|C^{1}\left(\mathfrak{B}_{a_{k}} \hookrightarrow \mathbb{S}_{k} ; n\right)\right| .
\end{aligned}
$$

By taking the $S_{n}$-action on the underlying sets of both sides of this equation, we have

$$
\operatorname{Isoc}\left(\mathbb{S}_{k}, B ; n\right)=(-1)^{b} \operatorname{Isoc}\left(\mathbb{S}_{k}, \emptyset ; n\right)+\sum_{t=0}^{b-1}(-1)^{t}\left(\begin{array}{l}
b \\
t
\end{array}\right) \operatorname{Isoc}\left(\mathfrak{B}_{a_{k}+b-t-1} ; n\right) .
$$


The number $\operatorname{Isoc}(G ; n)$ was computed for any graph $G$ and any natural number $n$ by Liskovets [15] and by the first two authors (see [11]) as a recursive formula, and the number $\operatorname{Isoc}\left(\mathbb{S}_{k}, \emptyset ; n\right)$ was computed for any $k$ and $n$ by Mednykh (see $\left.[17,18]\right)$. In fact, Mednykh computed the number of conjugacy classes of subgroups of index $n$ in the fundamental group $\pi_{1}\left(\mathbb{S}_{k}, *\right)$ of a surface $\mathbb{S}_{k}$ which is equal to the number $\operatorname{Isoc}\left(\mathbb{S}_{k}, \varnothing ; n\right)$.

For convenience, let $\mathfrak{P}(n)$ denote the set of all partitions of the natural number $n$ : that is, the set of ordered sequences $\left(n_{1}, n_{2}, \ldots, n_{\ell}\right)$ with $n_{1} \leqslant n_{2} \leqslant \cdots \leqslant n_{\ell}$ of natural numbers such that $n_{1}+n_{2}+\cdots+n_{\ell}=n$. For a partition $\mathfrak{p}$ of $n$, let $j_{k}(\mathfrak{p})$ denote the multiplicity of $k$ in the partition $\mathfrak{p}$, so that $j_{1}(\mathfrak{p})+2 j_{2}(\mathfrak{p})+\cdots+n j_{n}(\mathfrak{p})=n$. A partition $\mathfrak{p}$ of $n$ is denoted by $[[k ; n / k]]$ if every term of $\mathfrak{p}$ is $k$. Note that $[[k ; m]]$ denotes the partition of the natural number $k m$, each of whose terms is $k$.

THEOREM 3 (see [11]). For $n \geqslant 2$, the number of non-isomorphic connected $n$-fold coverings of $\mathfrak{B}_{m}$ is

$$
\begin{aligned}
& \operatorname{Isoc}\left(\mathfrak{B}_{m} ; n\right)=\sum_{\ell_{1}+2 \ell_{2}+\cdots+(n-1) \ell_{n-1}=n-1}\left(\left(\ell_{1}+1\right)^{m-1}-1\right) \\
& \times\left(\ell_{1} ! 2^{\ell_{2}} \ell_{2} ! \cdots(n-1)^{\ell_{n-1}} \ell_{n-1} !\right)^{m-1} \\
& +\sum_{2 \ell_{2}+3 \ell_{3}+\cdots+n \ell_{n}=n}\left(2^{\ell_{2}} \ell_{2} ! 3^{\ell_{3}} \ell_{3} ! \cdots n^{\ell_{n}} \ell_{n} !\right)^{m-1} \\
& -\sum_{\substack{\mathfrak{p} \in \mathfrak{P}(n)-\{[[n ; 1]]\} \\
j_{1}(\mathfrak{p})=0}} \prod_{\substack{j_{k}(\mathfrak{p}) \neq 0 \\
j_{k}(\mathfrak{p}) !}}\left(\prod_{\ell=0}^{j_{k}(\mathfrak{p})-1}\left(\operatorname{Isoc}\left(\mathfrak{B}_{m} ; k\right)+\ell\right)\right),
\end{aligned}
$$

where the summation over the empty index set is defined to be 0 .

THEOREM 4 (see $[17,18])$. The number of non-isomorphic connected $n$-fold unbranched coverings of a surface $\mathbb{S}_{k}$ of genus $k$ is

$\operatorname{Isoc}\left(\mathbb{S}_{k}, \emptyset ; n\right)= \begin{cases}\frac{1}{n} \sum_{m \mid n} \delta_{k}(m) \sum_{d \mid(n / m)} \mu\left(\frac{n}{m d}\right) d^{(2 k-2) m+2}, & \text { if } k \geqslant 0, \\ \frac{1}{n} \sum_{m \mid n} \sum_{d \mid(n / m)} \mu\left(\frac{n}{m d}\right) d^{(-k-2) m+1}\left[(2, d) \delta_{k}^{-}(m)+d \delta_{k}^{+}(m)\right], & \text { if } k<0,\end{cases}$

where $\wp_{k}(m)$ is the number of subgroups of index $m$ in the fundamental group $\pi_{1}\left(\mathbb{S}_{k}, *\right)$ of a surface $\mathbb{S}_{k}$ of genus $k, \mu$ is the Möbius function, $\delta_{k}^{+}(m)$ is equal to 0 if $m$ is odd and equal to $\wp_{k}(m / 2)$ if $m$ is even, $\wp_{k}^{-}(m)=\wp_{k}(m)-\wp_{k}^{+}(m)$, and $(2, d)$ is the greatest common divisor of 2 and $d$. In fact, the number $\wp_{k}(m)$ is given as follows:

$$
\S_{k}(m)=m \sum_{s=1}^{m} \frac{(-1)^{s+1}}{s} \sum_{\substack{i_{1}+i_{2}+\cdots+i_{s}=m \\ i_{1}, i_{2}, \ldots, i_{s} \geqslant 1}} \beta_{i_{1}} \beta_{i_{2}} \cdots \beta_{i_{s}},
$$

where

$$
\beta_{h}=\sum_{\chi \in D_{h}}\left(\frac{h !}{f(\chi)}\right)^{t}, \quad t= \begin{cases}2 k-2, & \text { if } k \geqslant 0, \\ -k-2, & \text { if } k<0 .\end{cases}
$$

$D_{h}$ is the set of all irreducible representations of the symmetric group $S_{h}$, and $f^{(\chi)}$ is the degree of the representation $\lambda$. 
As an illustration of Theorem 2, we compute explicitly the number of non-isomorphic 3 -fold branched coverings of the orientable surface $\mathbb{S}_{k}(k \geqslant 0)$ with branch set $B,(|B|=b)$.

It is already known that $\operatorname{Isoc}\left(\mathfrak{B}_{m} ; 3\right)=6^{m-1}+3^{m-1}-2^{m-1}$ (see [11]) and $\operatorname{Isoc}\left(\mathbb{S}_{k}, \emptyset ; 3\right)=2 \cdot 6^{2 k-2}+4 \cdot 3^{2 k-2}-2 \cdot 2^{2 k-2}$ (see [17]). Now, by applying Theorem 2 , we have

$$
\operatorname{Isoc}\left(\mathbb{S}_{k}, B ; 3\right)=6^{2 k-2}\left(5^{b}+(-1)^{b}\right)+3^{2 k-2}\left(2^{b}+(-1)^{b} 3\right)-2^{2 k-2}\left(1+(-1)^{b}\right) .
$$

\section{Regular coverings}

In this section, we aim to compute the number $\operatorname{Isoc}^{R}\left(\mathbb{S}_{k}, B ; n\right)$ of non-isomorphic connected regular $n$-fold branched coverings of a surface $\mathbb{S}_{k}$ with branch set $B$. Any two connected regular branched coverings are not isomorphic if their covering transformation groups (or voltage groups) are not isomorphic. Hence, the following equation comes from the fact that every connected regular $n$-fold branched covering is isomorphic to a connected branched $\mathcal{A}$-covering for some group $\mathcal{A}$ of order $n$ :

$$
\operatorname{Isoc}^{R}\left(\mathbb{S}_{k}, B ; n\right)=\sum_{\mathcal{A}} \operatorname{Isoc}\left(\mathbb{S}_{k}, B ; \mathcal{A}\right),
$$

where $\mathcal{A}$ runs over all the representatives of the isomorphism classes of groups of order $n$.

Now, we need to compute the number $\operatorname{Isoc}\left(\mathbb{S}_{k}, B ; \mathcal{A}\right)$ for each finite group $\mathcal{A}$. Let $\operatorname{Aut}(\mathcal{A})$ denote the group of automorphisms of $\mathcal{A}$, and we define an $\operatorname{Aut}(\mathcal{A})$-action on $C^{1}\left(\mathfrak{B}_{m} ; \mathcal{A}\right)$ as follows. For any $\sigma \in \operatorname{Aut}(\mathcal{A})$ and any $\left(g_{1}, \ldots, g_{m}\right) \in C^{1}\left(\mathfrak{B}_{m} ; \mathcal{A}\right)$, we define

$$
\sigma \cdot\left(g_{1}, \ldots, g_{m}\right)=\left(\sigma\left(g_{1}\right), \ldots, \sigma\left(g_{m}\right)\right) .
$$

Then it follows from Corollary 1 that two voltage assignments in $C^{1}\left(\mathfrak{B}_{a_{k}+|B|} \hookrightarrow \mathbb{S}_{k}-B ; \mathcal{A}\right)$ derive isomorphic branched coverings of $\mathbb{S}_{k}$ if and only if they belong to the same orbit under the $\operatorname{Aut}(\mathcal{A})$-action. Notice that this $\operatorname{Aut}(\mathcal{A})$-action on $C^{1}\left(\mathfrak{B}_{a_{k}+|B|} \hookrightarrow \mathbb{S}_{k}-B ; \mathcal{A}\right)$ is free because $\left\{g_{1}, \ldots, g_{a_{k}+b}\right\}$ generates $\mathcal{A}$. This implies that the number $\operatorname{Isoc}\left(\mathbb{S}_{k}, B ; \mathcal{A}\right)$ of non-isomorphic connected branched $\mathcal{A}$-coverings of the surface $\mathbb{S}_{k}$ with branch set $B$ is

$$
\operatorname{Isoc}\left(\mathbb{S}_{k}, B ; \mathcal{A}\right)=\frac{\left|C^{1}\left(\mathfrak{B}_{a_{k}+|B|} \hookrightarrow \mathbb{S}_{k}-B ; \mathcal{A}\right)\right|}{|\operatorname{Aut}(\mathcal{A})|} .
$$

By using a method similar to the proof of Theorem 2, one can derive the following theorem.

THEOREM 5. Let $k$ be any integer, and let $B$ be a b-subset of the surface $\mathbb{S}_{k}$. Then, for any finite group $\mathcal{A}$, the number of connected branched $\mathcal{A}$-coverings of the surface $\mathbb{S}_{k}$ with branch set $B$ is

$$
\operatorname{Isoc}\left(\mathbb{S}_{k}, B ; \mathcal{A}\right)=(-1)^{b} \operatorname{Isoc}\left(\mathbb{S}_{k}, \emptyset ; \mathcal{A}\right)+\sum_{t=0}^{b-1}(-1)^{t}\left(\begin{array}{l}
b \\
t
\end{array}\right) \operatorname{Isoc}\left(\mathfrak{B}_{a_{k}+b-t-1} ; \mathcal{A}\right),
$$

where $\mathfrak{B}_{m}$ is a bouquet of $m$ circles, $a_{k}=2 k$ if $k \geqslant 0$, and $a_{k}=-k$ if $k<0$.

To calculate the number of coverings in Theorem 5, we follow the method used by Jones in $[7,8]$. Let $\mathcal{F}=\pi_{1}\left(\mathbb{S}_{k}, *\right)$ be the fundamental group of the surface $\mathbb{S}_{k}$. Denote by $\operatorname{Hom}(\mathcal{F} ; \mathcal{A})$ and $\operatorname{Epi}(\mathcal{F} ; \mathcal{A})$, respectively, the set of homomorphisms and the set of epimorphisms of the group $\mathcal{F}$ into the group $\mathcal{A}$. 
Then one can see that $\left|C^{1}\left(\mathfrak{B}_{a_{k}} \hookrightarrow \mathbb{S}_{k} ; \mathcal{A}\right)\right|=|\operatorname{Epi}(\mathcal{F} ; \mathcal{A})|$, so that

$$
\operatorname{Isoc}\left(\mathbb{S}_{k}, \emptyset ; \mathcal{A}\right)=\frac{|\operatorname{Epi}(\mathcal{F} ; \mathcal{A})|}{|\operatorname{Aut}(\mathcal{A})|} .
$$

Also, we have

$$
|\operatorname{Hom}(\mathcal{F}, \mathcal{A})|=\sum_{K \leqslant \mathcal{A}}|\operatorname{Epi}(\mathcal{F}, K)|,
$$

where the sum is taken over all subgroups $K$ of the group $\mathcal{A}$. Now, one can invert this equation to count epimorphisms in terms of homomorphisms, by introducing the Möbius function for $\mathcal{A}$. This assigns an integer $\mu(K)$ to each subgroup $K$ of $\mathcal{A}$ by the recursive formula

$$
\sum_{H \geqslant K} \mu(H)=\delta_{K, \mathcal{A}}= \begin{cases}1, & \text { if } K=\mathcal{A}, \\ 0, & \text { if } K<\mathcal{A} .\end{cases}
$$

The equation

$$
|\operatorname{Epi}(\mathcal{F}, \mathcal{A})|=\sum_{K \leqslant \mathcal{A}} \mu(K)|\operatorname{Hom}(\mathcal{F}, K)|
$$

is then easily deduced. As a result we have the following lemma.

Lemma 3. Let $\mathcal{F}=\pi_{1}\left(\mathbb{S}_{k}, *\right)$ be the fundamental group of a surface $\mathbb{S}_{k}$. Then

$$
\operatorname{Isoc}\left(\mathbb{S}_{k}, \emptyset ; \mathcal{A}\right)=\frac{1}{|\operatorname{Aut}(\mathcal{A})|} \sum_{K \leqslant \mathcal{A}} \mu(K)|\operatorname{Hom}(\mathcal{F}, K)| .
$$

In a similar way, taking into account the fact that the fundamental group of the bouquet $\mathfrak{B}_{r}$ of $r$ circles $\pi_{1}\left(\mathfrak{B}_{r}\right)=\mathcal{F}_{r}$ is the free group of rank $r$, we obtain the next lemma.

Lemma 4. Let $\mathfrak{B}_{r}$ be a bouquet of $r$ circles. Then

$$
\begin{aligned}
\operatorname{Isoc}\left(\mathfrak{B}_{r} ; \mathcal{A}\right)=\frac{\left|\operatorname{Epi}\left(\mathcal{F}_{r}, \mathcal{A}\right)\right|}{|\operatorname{Aut}(\mathcal{A})|} & =\frac{1}{|\operatorname{Aut}(\mathcal{A})|} \sum_{K \leqslant \mathcal{A}} \mu(K)\left|\operatorname{Hom}\left(\mathcal{F}_{r}, K\right)\right| \\
& =\frac{1}{|\operatorname{Aut}(\mathcal{A})|} \sum_{K \leqslant \mathcal{A}} \mu(K)|K|^{r} .
\end{aligned}
$$

The number $\operatorname{Isoc}\left(\mathfrak{B}_{m} ; \mathcal{A}\right)$ has been explicitly computed for any $m$ and any finite abelian group $\mathcal{A}$ or any dihedral group $\mathbb{D}_{n}$ of order $2 n$ (see [13]). Notice that the formulae in [13] do not involve the lattice structure of subgroups of $\mathcal{A}$. When $\mathcal{A}$ is abelian the formula depends only on the decompositions of $\mathcal{A}$, and when $\mathcal{A}=\mathbb{D}_{n}$ the formula depends only on the prime decompositions of $n$.

Denote by $H_{\mathbb{S}_{k}}(\mathcal{A})=\left|\operatorname{Hom}\left(\pi_{1}\left(\mathbb{S}_{k}, *\right) ; \mathcal{A}\right)\right|$ the number of homomorphisms of the fundamental group $\pi_{1}\left(\mathbb{S}_{k}, *\right)$ into a finite group $\mathcal{A}$. The following result, essentially due to Frobenius, was given in different cases in [7], [9] and [17].

Lemma 5. (a) Let $\mathbb{S}_{k}$ be a closed orientable surface of genus $k \geqslant 0$. Then

$$
H_{\mathbb{S}_{k}}(\mathcal{A})=|\mathcal{A}|^{2 k-1} \sum_{\chi} \chi(1)^{2-2 k}
$$

where $\chi$ ranges over all irreducible characters of $\mathcal{A}$ and $\chi(1)$ is degree of $\chi$. 
(b) Let $\mathbb{S}_{k}$ be a closed non-orientable surface of genus $-k, k<0$. Then

$$
H_{\mathbb{S}_{k}}(\mathcal{A})=|\mathcal{A}|^{-k-1} \sum_{\chi} c_{\chi}^{-k} \chi(1)^{2+k}
$$

where, with the representation $\rho$ corresponding the character $\chi$,

$$
c_{\chi}=\frac{1}{|\mathcal{A}|} \sum_{g \in \mathcal{A}} \chi\left(g^{2}\right)=\left\{\begin{aligned}
+1, & \text { if } \rho \text { is real, } \\
-1, & \text { if } \chi \text { is real but } \rho \text { is not real, } \\
0, & \text { if } \chi \text { is not real, }
\end{aligned}\right.
$$

is the Frobenius-Schur indicator of $\rho$ and $\chi$.

By virtue of Lemmas 3 and 4, the statement of Theorem 5 can be rephrased as

$$
\operatorname{Isoc}\left(\mathbb{S}_{k}, B ; \mathcal{A}\right)=\frac{1}{|\operatorname{Aut}(\mathcal{A})|} \sum_{K \leqslant \mathcal{A}} \mu(K)\left((-1)^{b} H_{\mathbb{S}_{k}}(K)+\sum_{t=0}^{b-1}(-1)^{t}\left(\begin{array}{l}
b \\
t
\end{array}\right)|K|^{a_{k}+b-t-1}\right) \text {. }
$$

Applying Lemma 5, we obtain the following result.

TheORem 6. Let $B$ be a b-subset of a surface $\mathbb{S}_{k}$, and let $\mathcal{A}$ be a finite group. Then we have

$\operatorname{Isoc}\left(\mathbb{S}_{k}, B ; \mathcal{A}\right)$

$$
= \begin{cases}\sum_{K \leqslant A} \frac{\mu(K)|K|^{2 k-1}}{|\operatorname{Aut}(\mathcal{A})|}\left((|K|-1)^{b}+(-1)^{b} \sum_{\chi} \chi(1)^{2-2 k}\right), & \text { if } k \geqslant 0, \\ \sum_{K \leqslant A} \frac{\mu(K)|K|^{-k-1}}{|\operatorname{Aut}(\mathcal{A})|}\left((|K|-1)^{b}+(-1)^{b} \sum_{\chi} c_{\chi}^{-k} \chi(1)^{2+k}\right), & \text { if } k<0,\end{cases}
$$

where $\chi$ ranges over all irreducible characters of $K$ except the principal one and

$$
c_{\chi}=\frac{1}{|\mathcal{A}|} \sum_{g \in \mathcal{A}} \chi\left(g^{2}\right)=\left\{\begin{aligned}
+1, & \text { if } \rho \text { is real, } \\
-1, & \text { if } \chi \text { is real but } \rho \text { is not real, } \\
0, & \text { if } \chi \text { is not real, }
\end{aligned}\right.
$$

with the representation $\rho$ corresponding to $\chi$.

Let $\mathcal{A}$ be a finite abelian group, and let $\lambda(\mathcal{A})$ denote the number of direct summands of $\mathcal{A}$ whose orders are even. Since every character of an abelian group is linear and every irreducible character of an abelian group can be obtained by the product of irreducible characters of its direct summands, the number of irreducible real characters of an abelian group $\mathcal{A}$ is equal to $2^{\lambda(\mathcal{A})}$. For example, $\lambda\left(\mathbb{Z}_{6} \oplus \mathbb{Z}_{8}\right)=2$, and hence $\mathbb{Z}_{6} \oplus \mathbb{Z}_{8}$ has four irreducible real characters. Now we have the following corollary.

Corollary 2. Let $B$ be a $b$-subset of $\mathbb{S}_{k}$, and let $\mathcal{A}$ be a finite abelian group. Then we have

$$
\operatorname{Isoc}\left(\mathbb{S}_{k}, B ; \mathcal{A}\right)= \begin{cases}\sum_{K \leqslant A} \frac{\mu(K)|K|^{2 k-1}}{|\operatorname{Aut}(\mathcal{A})|}\left((|K|-1)^{b}+(-1)^{b}(|K|-1)\right), & \text { if } k \geqslant 0, \\ \sum_{K \leqslant A} \frac{\mu(K)|K|^{-k-1}}{|\operatorname{Aut}(\mathcal{A})|}\left((|K|-1)^{b}+(-1)^{b}\left(2^{\lambda(K)}-1\right)\right), & \text { if } k<0,\end{cases}
$$

where $\lambda(K)$ is the number of direct summands of $\mathcal{A}$ whose orders are even. 
We observe that Theorem 6 and Corollary 2 are quite efficient if the lattice structure of subgroups of $\mathcal{A}$, and their characters, are known. However, even though $\mathcal{A}$ is abelian, it is not easy to use Corollary 2 for $\operatorname{Isoc}\left(\mathbb{S}_{k}, B ; \mathcal{A}\right)$ if $|\mathcal{A}|$ is so large that the lattice structure of its subgroups is complicated.

In the next section we shall give another enumeration formula, for use when $\mathcal{A}$ is abelian, which does not involve the lattice structure of subgroups of $\mathcal{A}$.

\section{More on abelian branched coverings}

In this section, we aim to derive an explicit enumeration formula for $\operatorname{Isoc}\left(\mathbb{S}_{k}, B ; \mathcal{A}\right)$ when $\mathcal{A}$ is abelian. It is possible to obtain this from Theorem 5 , by deriving a computational formula for $\operatorname{Isoc}\left(\mathbb{S}_{k}, \emptyset ; \mathcal{A}\right)$ that does not involve the lattice structure of subgroups of $\mathcal{A}$ when $\mathcal{A}$ is abelian.

Let $\mathcal{A}=\mathcal{A}_{1} \oplus \mathcal{A}_{2}$ with $\left(\left|\mathcal{A}_{1}\right|,\left|\mathcal{A}_{2}\right|\right)=1$. Then

$$
\left|C^{1}\left(\mathfrak{B}_{a_{k}} \hookrightarrow \mathbb{S}_{k} ; \mathcal{A}\right)\right|=\left|C^{1}\left(\mathfrak{B}_{a_{k}} \hookrightarrow \mathbb{S}_{k} ; \mathcal{A}_{1}\right)\right| \cdot\left|C^{1}\left(\mathfrak{B}_{a_{k}} \hookrightarrow \mathbb{S}_{k} ; \mathcal{A}_{2}\right)\right|
$$

and $|\operatorname{Aut}(\mathscr{A})|=\left|\operatorname{Aut}\left(\mathscr{A}_{1}\right)\right| \cdot\left|\operatorname{Aut}\left(\mathscr{A}_{2}\right)\right|$. Hence we have the following lemma.

LeMma 6. For any finite groups $\mathcal{A}$ and $\mathscr{B}$ with $(|\mathcal{A}|,|\mathscr{B}|)=1$, and for any surface $\mathbb{S}_{k}$, we have

$$
\operatorname{Isoc}\left(\mathbb{S}_{k}, \emptyset ; \mathcal{A} \oplus \mathscr{B}\right)=\operatorname{Isoc}\left(\mathbb{S}_{k}, \emptyset ; \mathcal{A}\right) \cdot \operatorname{Isoc}\left(\mathbb{S}_{k}, \emptyset ; \mathscr{B}\right)
$$

Let $\mathcal{A}$ be an abelian group. If $k \geqslant 0$ and $B=\varnothing$, then conditions (C2) and (C3) in the definition of the set $C^{1}\left(\mathfrak{B}_{a_{k}} \hookrightarrow \mathbb{S}_{k} ; \mathcal{A}\right)$ are clearly satisfied, so that $\operatorname{Isoc}\left(\mathbb{S}_{k} ; \emptyset ; \mathcal{A}\right)=$ $\operatorname{Isoc}\left(\mathfrak{B}_{2 k} ; \mathcal{A}\right)$, as has already been computed in [13]. If $k<0$ and $B=\emptyset$, then $C^{1}\left(\mathfrak{B}_{a_{k}} \hookrightarrow\right.$ $\left.\mathbb{S}_{k} ; \mathcal{A}\right)$ is equal to the set of $(-k)$-tuples $\left(g_{1}, \ldots, g_{-k}\right)$ with the properties that $\left\{g_{1}, \ldots, g_{-k}\right\}$ generates $\mathcal{A}$ and $\left(g_{1}\right)^{2} \cdots\left(g_{-k}\right)^{2}=1$. For convenience, let

$$
\begin{aligned}
& \mathfrak{F}\left(\mathfrak{B}_{-k} ; \mathcal{A}\right) \\
& \quad=\left\{\left(g_{1}, g_{2}, \ldots, g_{-k}\right) \in \mathcal{A}^{-k}:\left\{g_{1}, g_{2}, \ldots, g_{-k}\right\} \text { generates } \mathcal{A} \text { and }\left(g_{-k}\right)^{2}=1\right\} .
\end{aligned}
$$

Define a function $f: C^{1}\left(\mathfrak{B}_{a_{k}} \hookrightarrow \mathbb{S}_{k} ; \mathcal{A}\right) \rightarrow \mathfrak{F}\left(\mathfrak{B}_{-k} ; \mathcal{A}\right)$ by

$$
f\left(g_{1}, \ldots, g_{-k}\right)=\left(g_{1}, \ldots, g_{-k-1}, g_{1} \cdots g_{-k}\right) .
$$

Then $f$ is well defined, because $\left(g_{1}\right)^{2} \cdots\left(g_{-k}\right)^{2}=\left(g_{1} \cdots g_{-k}\right)^{2}$ in the abelian group $\mathcal{A}$. Now, it is not hard to see that $f$ is a bijection. Hence we have

$$
\operatorname{Isoc}\left(\mathbb{S}_{k}, \emptyset ; \mathcal{A}\right)=\frac{\left|C^{1}\left(\mathfrak{B}_{a_{k}} \hookrightarrow \mathbb{S}_{k} ; \mathcal{A}\right)\right|}{|\operatorname{Aut}(\mathcal{A})|}=\frac{\left|\mathfrak{F}\left(\mathfrak{B}_{-k} ; \mathcal{A}\right)\right|}{|\operatorname{Aut}(\mathcal{A})|} .
$$

By the classification theorem of finite abelian groups, one can express a finite abelian group $\mathcal{A}$ as follows.

$$
\mathcal{A}=\mathcal{A}_{o} \oplus \mathcal{A}_{e}=\left(\oplus_{i=1}^{S} \oplus_{j=1}^{t_{i}} m_{i_{j}} \mathbb{Z}_{p_{i}} \ell_{i_{j}}\right) \bigoplus\left(\oplus_{k=1}^{\ell} m_{k} \mathbb{Z}_{2^{t_{k}}}\right),
$$

where $p_{i}$ are odd primes and $p_{i} \neq p_{i^{\prime}}$ if $i \neq i^{\prime}$; that is, $\mathcal{A}_{e}$ is the subgroup of $\mathcal{A}$ consisting of all elements of order a power of 2 . Let $\theta(\mathcal{A})$ denote the number of direct summands of $\mathcal{A}$ whose orders are multiples of 4 , and let $\omega(\mathcal{A})$ denote the number of direct summands of $\mathcal{A}$ whose orders are 2 . Notice that $\lambda(\mathcal{A})=\theta(\mathcal{A})+\omega(\mathcal{A})$. For example, $\mathbb{Z}_{8} \oplus \mathbb{Z}_{10}=$ $\mathbb{Z}_{8} \oplus \mathbb{Z}_{2} \oplus \mathbb{Z}_{5}, \theta\left(\mathbb{Z}_{8} \oplus \mathbb{Z}_{10}\right)=1$ and $\omega\left(\mathbb{Z}_{8} \oplus \mathbb{Z}_{10}\right)=1$. 
Clearly, $\left(\left|\mathcal{A}_{o}\right|,\left|\mathcal{A}_{e}\right|\right)=1$ for any abelian group $\mathcal{A}$ and, by Lemma 6,

$$
\operatorname{IsOc}\left(\mathbb{S}_{k}, \emptyset ; \mathcal{A}\right)=\operatorname{IsOc}\left(\mathbb{S}_{k}, \varnothing ; \mathcal{A}_{o}\right) \cdot \operatorname{IsOc}\left(\mathbb{S}_{k}, \emptyset ; \mathcal{A}_{e}\right) .
$$

Note that the order of $\mathcal{A}_{o}$ is odd. In an abelian group of odd order, $g^{2}=1$ implies that $g=1$, and hence $\mathfrak{F}\left(\mathfrak{B}_{-k} ; \mathcal{A}_{0}\right)=\left|\mathfrak{C}\left(\mathfrak{B}_{-k-1} ; \mathcal{A}_{0}\right)\right|$. Now, using Lemma 6 and a computational method similar to [13, Lemma 3.3], one can obtain

$$
\begin{aligned}
& \left|\mathfrak{F}\left(\mathfrak{B}_{-k} ; \mathcal{A}\right)\right| \\
& =\frac{2^{-k}-2^{\theta(\mathcal{A})}}{\left(2^{-k}-1\right) 2^{\sum_{k=1}^{\ell} m_{k}\left(t_{k}-1\right)}}\left|\mathfrak{C}\left(\mathfrak{B}_{-k-1} ; \mathcal{A}_{o}\right)\right|\left|\mathfrak{C}\left(\mathfrak{B}_{-k} ; \mathcal{A}_{e}\right)\right|
\end{aligned}
$$

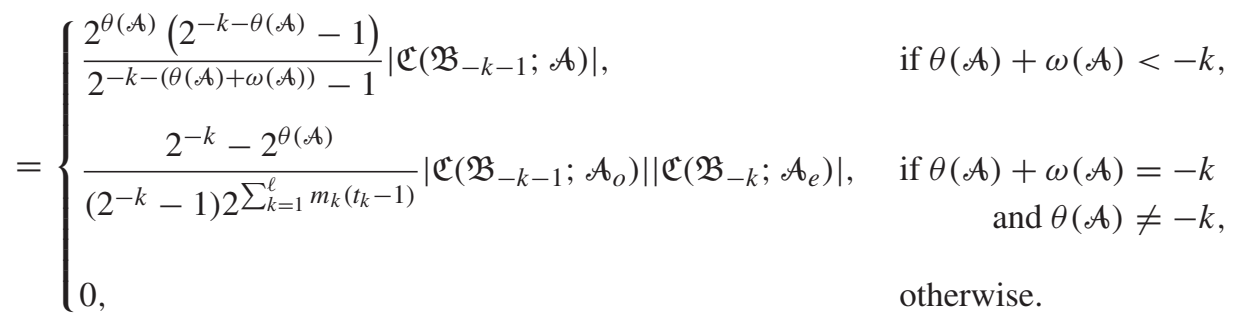

We summarize our discussions as follows.

Lemma 7. Let $\mathbb{S}_{k}$ be a surface of genus $k$, and let $\mathcal{A}$ be any finite abelian group. Then the following statements hold.

(a),$k \geqslant 0$, then $\operatorname{Isoc}\left(\mathbb{S}_{k}, \emptyset ; \mathcal{A}\right)=\operatorname{Isoc}\left(\mathfrak{B}_{2 k} ; \mathcal{A}\right)$.

(b) If $k<0$, then

$\operatorname{IsOc}\left(\mathbb{S}_{k}, \emptyset ; \mathcal{A}\right)$

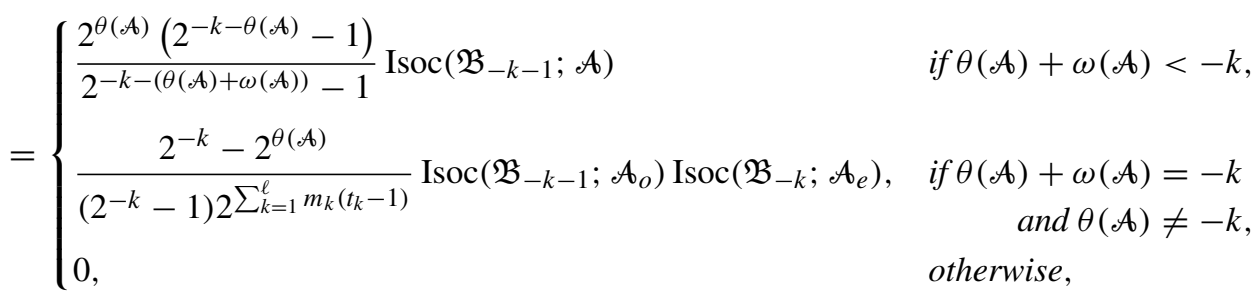

where $\mathcal{A}=\mathcal{A}_{o} \oplus \mathcal{A}_{e}=\left(\oplus_{i=1}^{S} \oplus_{j=1}^{t_{i}} m_{i_{j}} \mathbb{Z}_{p_{i} \ell_{i_{j}}}\right) \bigoplus\left(\oplus_{k=1}^{\ell} m_{k} \mathbb{Z}_{2^{t_{k}}}\right)$.

COROLlary 3. Let $\mathbb{S}_{k}$ be any nonorientable surface, and let $\mathcal{A}$ be any finite abelian group. Then the following statements hold.

(a) If $\mathcal{A}$ does not contain $\mathbb{Z}_{2}$ as its direct summand, then

$$
\operatorname{Isoc}\left(\mathbb{S}_{k}, \varnothing ; \mathcal{A}\right)= \begin{cases}2^{\theta(\mathcal{A})} \operatorname{Isoc}\left(\mathfrak{B}_{-k-1} ; \mathcal{A}\right), & \text { if } \theta(\mathcal{A})<-k, \\ 0, & \text { otherwise. }\end{cases}
$$

In particular, if the order of $\mathcal{A}$ is odd, then $\operatorname{Isoc}\left(\mathbb{S}_{k}, \emptyset ; \mathcal{A}\right)=\operatorname{Isoc}\left(\mathfrak{B}_{-k-1} ; \mathcal{A}\right)$.

(b) If $\mathcal{A}$ is $m \mathbb{Z}_{2}$, then $\operatorname{Isoc}\left(\mathbb{S}_{k}, \emptyset ; m \mathbb{Z}_{2}\right)=\operatorname{Isoc}\left(\mathfrak{B}_{-k} ; m \mathbb{Z}_{2}\right)$. 


\section{Explicit enumeration of some regular coverings}

Recall that one can explicitly enumerate the total number Isoc $\left(\mathbb{S}_{k}, B ; n\right)$ of connected $n$-fold branched coverings of a surface by Theorem 2 , but it is not easy to enumerate explicitly the number $\operatorname{Isoc}^{R}\left(\mathbb{S}_{k}, B ; n\right)$ of regular $n$-fold branched surface coverings of a given surface $\mathbb{S}_{k}$ with branch set $B$. In this section, we compute the number $\operatorname{Isoc}^{R}\left(\mathbb{S}_{k}, B ; p\right)$, $\operatorname{Isoc}^{R}\left(\mathbb{S}_{k}, B ; 2 p\right)$ or $\operatorname{Isoc}^{R}\left(\mathbb{S}_{k}, B ; p^{2}\right)$ for any prime number $p$, as possible cases.

First, we compute $\operatorname{Isoc}^{R}\left(\mathbb{S}_{k}, B ; p\right)$ for any prime $p$. It is already known [13] that for any prime $p, \operatorname{Isoc}\left(\mathfrak{B}_{m} ; \mathbb{Z}_{p}\right)=\left(p^{m}-1\right) /(p-1)$. Since every group of order $p$ is isomorphic to the cyclic group $\mathbb{Z}_{p}$, it follows that $\operatorname{Isoc}^{R}\left(\mathbb{S}_{k}, B ; p\right)=\operatorname{Isoc}\left(\mathbb{S}_{k}, B ; \mathbb{Z}_{p}\right)$ for any $k$ and any finite subset $B$ of $\mathbb{S}_{k}$. Now, by applying Theorem 5, Lemma 7 and Corollary 3, one can obtain the following theorem.

THEOREM 7. Let $B$ be a $b$-subset of a surface $\mathbb{S}_{k}$, and let $p$ be a prime. Then the number $\operatorname{Isoc}^{R}\left(\mathbb{S}_{k}, B ; p\right)$ of non-isomorphic regular connected branched p-fold coverings of $\mathbb{S}_{k}$ with branch set $B$ is

$$
\operatorname{Isoc}^{R}\left(\mathbb{S}_{k}, B ; p\right)= \begin{cases}\frac{p^{2 k}-1}{p-1}, & \text { if } k \geqslant 0 \text { and } b=0, \\ p^{2 k-1}\left((p-1)^{b-1}+(-1)^{b}\right), & \text { if } k \geqslant 0 \text { and } b \neq 0, \\ 2^{-k}-1, & \text { if } k<0, b=0 \text { and } p=2, \\ \frac{2^{-k-1}\left(1+(-1)^{b}\right),}{p-1}, & \text { if } k<0, b \neq 0 \text { and } p=2, \\ p^{-k-1}(p-1)^{b-1}, & \text { if } k<0, b=0 \text { and } p \neq 2,\end{cases}
$$

Notice that $\operatorname{Isoc}^{R}\left(\mathbb{S}_{k}, B ; p\right)$ has already been computed in [7], [14] and [16], but the computational methods used there are different from that given in this paper.

Next, to compute $\operatorname{Isoc}^{R}\left(\mathbb{S}_{k}, B ; p^{2}\right)$ for any prime $p$, recall that every finite group of order $p^{2}$ is abelian and is isomorphic to $\mathbb{Z}_{p^{2}}$ or $\mathbb{Z}_{p} \times \mathbb{Z}_{p}$. It is known [13] that for any prime $p$,

$$
\operatorname{Isoc}\left(\mathfrak{B}_{m} ; \mathbb{Z}_{p^{2}}\right)=\frac{p^{2 m-1}-p^{m-1}}{p-1}
$$

and

$$
\operatorname{Isoc}\left(\mathfrak{B}_{m} ; \mathbb{Z}_{p} \oplus \mathbb{Z}_{p}\right)=\frac{p^{2 m-1}-p^{m-1}(p+1)+1}{\left(p^{2}-1\right)(p-1)}
$$

By Lemma 7 and Corollary 3, we can have

$$
\operatorname{Isoc}\left(\mathbb{S}_{k}, \varnothing ; \mathbb{Z}_{p^{2}}\right)= \begin{cases}\frac{p^{4 k-1}-p^{2 k-1}}{p-1}, & \text { if } k \geqslant 0, \\ 2^{-2 k-2}-2^{-k-1}, & \text { if } k<0 \text { and } p=2, \\ \frac{p^{-2 k-3}-p^{-k-2}}{p-1}, & \text { if } k<0 \text { and } p \neq 2,\end{cases}
$$


and

$$
\operatorname{Isoc}\left(\mathbb{S}_{k}, \emptyset ; \mathbb{Z}_{p} \times \mathbb{Z}_{p}\right)= \begin{cases}\frac{p^{4 k-1}-p^{2 k-1}(p+1)+1}{\left(p^{2}-1\right)(p-1)}, & \text { if } k \geqslant 0, \\ \frac{2^{-2 k-1}-3 \cdot 2^{-k-1}+1}{3}, & \text { if } k<0 \text { and } p=2, \\ \frac{p^{-2 k-3}-p^{-k-2}(p+1)+1}{\left(p^{2}-1\right)(p-1)}, & \text { if } k<0 \text { and } p \neq 2 .\end{cases}
$$

By using these formulas and Theorem 5, we have the following theorem.

THEOREM 8. Let $B$ be a b-subset of a surface $\mathbb{S}_{k}$. Then the number $\operatorname{Isoc}^{R}\left(\mathbb{S}_{k}, B ; 4\right)$ of nonisomorphic regular connected branched 4-fold coverings of $\mathbb{S}_{k}$ with branch set $B$ is

$$
\begin{aligned}
& \operatorname{Isoc}^{R}\left(\mathbb{S}_{k}, B ; 4\right)= \\
& \begin{cases}(1 / 3)\left(2^{4 k+1}+1\right)-2^{2 k}, & \text { if } k \geqslant 0 \text { and } b=0, \\
2^{2 k-1}\left[2^{2 k}\left(3^{b-1}+(-1)^{b}\right)-\left(1+(-1)^{b}\right)\right], & \text { if } k \geqslant 0 \text { and } b \neq 0, \\
(1 / 3)\left(5 \cdot 2^{-2(k+1)}+1\right)-2^{-k}, & \text { if } k<0 \text { and } b=0, \\
2^{-k-1}\left[2^{-k-1}\left(2 \cdot 3^{b-1}+(-1)^{b}\right)-\left(1+(-1)^{b}\right)\right], & \text { if } k<0 \text { and } b \neq 0 .\end{cases}
\end{aligned}
$$

THEOREM 9. Let $B$ be a b-subset of a surface $\mathbb{S}_{k}$, and let $p$ be an odd prime. Then the number $\operatorname{Isoc}^{R}\left(\mathbb{S}_{k}, B ; p^{2}\right)$ of non-isomorphic regular connected branched $p^{2}$-fold coverings of $\mathbb{S}_{k}$ with branch set $B$ is

$$
\begin{aligned}
& \operatorname{Isoc}^{R}\left(\mathbb{S}_{k}, B ; p^{2}\right) \\
& = \begin{cases}\frac{\left(p^{2 k+1}-1\right)\left(p^{2 k}-1\right)}{\left(p^{2}-1\right)(p-1)}, & \text { if } k \geqslant 0 \text { and } b=0, \\
\frac{p^{2 k-1}}{p-1}\left[(p-1)^{b-1}\left(p^{2 k}(p+1)^{b-1}-1\right)+(-1)^{b}\left(p^{2 k}-1\right)\right], & \text { if } k \geqslant 0 \text { and } b \neq 0, \\
\frac{\left(p^{-k}-1\right)\left(p^{-k-1}-1\right)}{\left(p^{2}-1\right)(p-1)}, & \text { if } k<0 \text { and } b=0, \\
p^{-k-1}(p-1)^{b-2}\left[p^{-k}(p+1)^{b-1}-1\right], & \text { if } k<0 \text { and } b \neq 0 .\end{cases}
\end{aligned}
$$

Finally, we compute $\operatorname{Isoc}^{R}\left(\mathbb{S}_{k}, B ; 2 p\right)$ for any odd prime $p$. Recall that every finite group of order $2 p$ (where $p$ is an odd prime) is isomorphic to the cyclic group $\mathbb{Z}_{2 p}=\mathbb{Z}_{2} \times \mathbb{Z}_{p}$ or the dihedral group $\mathbb{D}_{p}$. It is known [13] that for any odd prime $p$,

$$
\operatorname{Isoc}\left(\mathfrak{B}_{m} ; \mathbb{Z}_{2 p}\right)=\frac{(2 p)^{m}-p^{m}-2^{m}+1}{p-1}
$$

and

$$
\operatorname{Isoc}\left(\mathfrak{B}_{m} ; \mathbb{D}_{p}\right)=\frac{2 \cdot(2 p)^{m-1}-p^{m-1}-2^{m}+1}{p-1}
$$


By Lemmas 6 and 7, and Corollary 3, one can have

$$
\operatorname{Isoc}\left(\mathbb{S}_{k}, \emptyset ; \mathbb{Z}_{2 p}\right)= \begin{cases}\frac{(2 p)^{2 k}-p^{2 k}-2^{2 k}+1}{p-1}, & \text { if } k \geqslant 0, \\ \frac{2 \cdot(2 p)^{-k-1}-p^{-k-1}-2^{-k}+1}{p-1}, & \text { if } k<0 .\end{cases}
$$

The number $\operatorname{Isoc}\left(\mathbb{S}_{k}, \emptyset ; \mathbb{D}_{p}\right)$ is already known, as follows (see [12]):

$$
\operatorname{Isoc}\left(\mathbb{S}_{k}, \varnothing ; \mathbb{D}_{p}\right)= \begin{cases}\frac{4 \cdot(2 p)^{2 k-2}-p^{2 k-2}-4 \cdot 2^{2 k-2}+1}{p-1}, & \text { if } k \geqslant 0, \\ \frac{4 \cdot(2 p)^{-k-2}+p^{-k-2}(p-2)-2^{-k}+1}{p-1}, & \text { if } k<0 .\end{cases}
$$

Now, the following theorem follows from these facts and Theorem 5.

THEOREM 10. Let $B$ be a $b$-subset of a surface $\mathbb{S}_{k}$ and let $p$ be an odd prime. Then the number $\operatorname{Isoc}^{R}\left(\mathbb{S}_{k}, B ; 2 p\right)$ of non-isomorphic regular connected branched $2 p$-fold coverings of $\mathbb{S}_{k}$ with branch set $B$ is

$$
\begin{aligned}
& \operatorname{Isoc}^{R}\left(\mathbb{S}_{k}, B ; 2 p\right) \\
& = \begin{cases}\frac{1}{p-1}\left(2^{2 k}-1\right)\left(\left(p^{2}+1\right) p^{2 k-2}-2\right), & \text { if } k \geqslant 0 \text { and } b=0, \\
\frac{2^{2 k-1} p^{2 k-2}}{p-1}\left[(2 p-1)^{b}(p+1)+(-1)^{b}\left(2 p^{2}-p+1\right)\right] & \\
-\frac{2^{2 k}\left(1+(-1)^{b}\right)}{p-1}-p^{2 k-2}\left[(p-1)^{b-1}(p+1)+(-1)^{b} p\right], & \text { if } k \geqslant 0 \text { and } b \neq 0, \\
\frac{1}{p-1}\left[2^{-k}\left(p^{-k-1}-1\right)+2\left(p^{-k-2}-1\right)\left(2^{-k-1}-1\right)\right], & \text { if } k<0 \text { and } b=0, \\
\frac{2^{-k-1} p^{-k-2}\left[\left((2 p-1)^{b}+(-1)^{b}\right)(p+1)\right]}{p-1}\left[\left(1+(-1)^{b}\right)\right. & \\
-\frac{2^{-k}\left(1+p^{-k-2}\left[(p-1)^{b-1}(p+1)-(-1)^{b}\right],\right.}{p-1} & \text { if } k<0 \text { and } b \neq 0 .\end{cases}
\end{aligned}
$$

\section{References}

1. I. BERSTEIN and A. L. EDMONDS, 'On the construction of branched coverings of lowdimensional manifolds', Trans. Amer. Math. Soc. 247 (1979) 87-124.

2. I. BERSTEIN and A. L. EDMONDS, 'On the classification of generic branched coverings of surfaces', Illinois J. Math. 28 (1984) 64-82.

3. J. L. Gross and T. W. TuCKER, 'Generating all graph coverings by permutation voltage assignments', Discrete Math. 18 (1977) 273-283. 90

4. J. L. Gross and T. W. Tucker, Topological graph theory (Wiley, New York, 1987). 91

5. U. HiRsch, 'On regular homotopy of branched coverings of the sphere', Manuscripta Math. 21 (1977) 293-306. 
6. A. HuRwitz, 'Uber Riemann'sche Flächen mit gegebenen Verzweigungspunkten', Math. Ann. 39 (1891) 1-60.

7. G. A. Jones, 'Enumeration of homomorphisms and surface-coverings', Quart. J. Math. Oxford (2) 46 (1995) 485-507. 96, 97, 101

8. G. A. Jones, 'Counting subgroups of non-Euclidean crystallographic groups', Math. Scand. 84 (1999) 23-39. 96

9. A. Kerber and B. Wegner, 'Gleichungen in endlichen Gruppen', Arch. Math. 35I (1980) 252-262. 97

10. J. H. KWAK and J. LeE, 'Isomorphism classes of graph bundles', Canad. J. Math. XLII (1990) 747-761. 93

11. J. H. KWAK and J. LEE, 'Enumeration of connected graph coverings', J. Graph Theory 23 (1996) 105-109. 93, 95, 96

12. J. H. KWAK and J. LEE, 'Distribution of branched $\mathbb{D}_{p}$-coverings of surfaces', Discrete Math. 183 (1998) 193-212. 89, 103

13. J. H. KWAK, J. CHUN and J. LEE, 'Enumeration of regular graph coverings having finite abelian covering transformation groups', SIAM J. Discrete Math. 11 (1998) 273-285. 97, 99, 100, 101, 102

14. J. H. KWAK, S. KIM and J. LEE, 'Distribution of regular branched prime-fold coverings of surfaces', Discrete Math. 156 (1996) 141-170. 89, 92, 101

15. V. Liskovets, 'Towards the enumeration of subgroups of the free group', Dokl. Akad. Nauk BSSR 15 (1971) 6-9 (in Russian). 95

16. A. D. MEDNYKh, 'Determination of the number of nonequivalent coverings over a compact Riemann surface', Soviet Math. Dokl. 19 (1978) 318-320. 101

17. A. D. MedNyKh, 'On unramified coverings of compact Riemann surfaces', Soviet Math. Dokl. 20 (1979) 85-88. 95, 96, 97

18. A. D. Mednykh and G. G. Pozdnyakova, 'Number of nonequivalent coverings over a nonorientable compact surface', Siber. Math. J. 27 (1986) 99-106. 95

19. S. Stahl, 'Generalized embedding schemes', J. Graph Theory 2 (1978) 41-52. 90

20. A. T. White, Graphs, groups and surfaces (North-Holland, N. York, 1984). 90

Jin Ho Kwak jinkwak@postech.ac.kr

Combinatorial and Computational Mathematics Center

Pohang University of Science and Technology

Pohang, 790-784, Korea

Jaeun Lee julee@yu.ac.kr

Mathematics

Yeungnam University

Kyongsan, 712-749, Korea

Alexander Mednykh mednykh@math.nsc.ru

Institute of Mathematics

Novosibirsk State University

Novosibirsk, 630090, Russia 